\title{
Estimación poblacional de Crocodylus moreletii en cuatro sitios de la Reserva de Biósfera Pantanos de Centla
}

\section{Population estimate of Crocodylus moreletii in four sites of the Pantanos de Centla Biosphere Reserve}

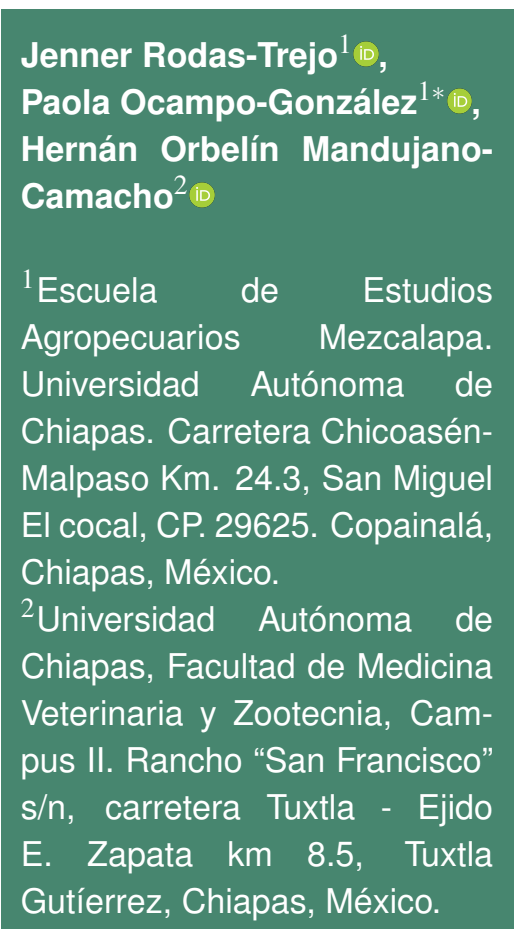

*Autor de correspondencia: paola.ocampo@unach.mx

Artículo científico

Recibido: 01 de julio 2020

Aceptado: 15 de octubre 2021

Como citar: Rodas-Trejo

J, Ocampo-González

Mandujano-Camacho

(2021) Estimación poblacional

de Crocodylus moreletii en

cuatro sitios de la Reserva

de Biósfera Pantanos de

Centla. Ecosistemas y

Recursos Agropecuarios

Núm. Esp. I: e2663. DOI:

10.19136/era.a8nl.2663
RESUMEN. La cuantificación de los cocodrilos en ambientes naturales representa información básica para establecer estrategias de manejo y conservación de estas especies. Con el objetivo de evaluar la tasa de encuentro (TE), abundancia y estructura poblacional del cocodrilo de pantano (Crocodylus moreletii) en la Reserva de la Biósfera Pantanos de Centla (RBPC), entre los meses de septiembre a diciembre de 2018 se llevaron a cabo recorridos mensuales nocturnos en las localidades Tabasquillo, Tembladeras, La Mixteca y Lázaro Cárdenas. En total se obtuvieron 797 registros en 16 conteos nocturnos. En la localidad de Tembladeras se registró la menor TE y tamaño poblacional con 1.12 cocodrilos $\mathrm{km}^{-1}$ lineal y $54.09 \pm 6.31$ individuos, respectivamente, en tanto que los valores más altos se presentaron en la localidad La Mixteca con 3.14 cocodrilos $\mathrm{km}^{-1}$ lineal y 84.79 \pm 6.44 individuos. Se encontraron diferencias significactivas entre los individuos observados por transecto $(H=10.775, g l .=3, p<0.05)$. En las cuatro localidades se observaron individuos de todas las clases de tamaño. Las mayores proporciones se presentaron en las clases I y II. Esta información contribuye al conocimiento sobre la ecología poblacional y es una primera aproximación el estado actual del cocodrilo de pantano y representa el primer estudio para $C$. moreletii en estas localidades de la RBPC.

Palabras clave: Abundancia, conteo, estructura poblacional, monitoreo, Tabasco.

ABSTRACT. The quantification of crocodiles in natural environments represents basic information to establish management and conservation strategies for these species. With the aim to estimate the encounter rate (ER), abundance and population structure of Morelet's Crocodile (Crocodylus moreletii) in the Pantanos de Centla Biosphere Reserve (PCBR), between the months of September to December 2018, they were carried out monthly night tours in the localities Tabasquillo, Tembladeras, La Mixteca and Lázaro Cárdenas. In total, 797 records were obtained in 16 night counts. In Tembladeras locality, the lowest ER and population size were recorded with 1.12 crocodiles linear $\mathrm{km}^{-1}$ and $54.09 \pm 6.31$ individuals, respectively, while the highest values are shown in La Mixteca locality with 3.14 crocodiles linear $\mathrm{km}^{-1}$ and $84.79 \pm 6.44$ individuals. Significant differences were found between the individuals observed by transect $(H=14.3457$, gl. $=3, p<$ 0.01 ). Individuals of all size classes were observed at all four locations. The highest proportions were presented in classes I and II. This information contributes to the knowledge about population ecology and a first approximation of the current state of the swamp crocodile and represents the first study for $C$. moreletii in this locaties of the PCBR.

Key words: Abundance, count, Population structure, monitoring, Tabasco. 


\section{INTRODUCCIÓN}

Los cocodrilos son especies que representan un recurso importante para las comunidades, ya que son utilizados como recurso alimenticio (BalagueraReina y González-Maya 2010), medicinal (CupulMagaña 2003, Rodas-Trejo et al. 2018), religioso (Barrera y Toledo 2005) y económico, principalmente por la venta de sus pieles (Casas-Andreu 2011). Sin embargo, debido a la caza ilegal y destrucción de su hábitat, sus poblaciones silvestres se vieron mermadas llevando a varias especies al borde de la extinción (Álvarez del Toro 1974).

El cocodrilo de pantano (Crocodylus moreletii), es una de las tres especies de cocodrilos que se encuentran en México (Álvarez del Toro 1974, Sigler y Gallegos 2017), su distribución en México abarca desde el estado de Tamaulipas, humedales de la planicie costera del Golfo de México, hasta la península de Yucatán y los países de Guatemala y Belice (Cedeño y Pérez 2010, López-Luna et al. 2019), habitando principalmente cuerpos de agua de caudal tranquilo (Álvarez del Toro, 1974). La población de $C$. moreletii está catalogada en el apéndice II de la Convención para el Comercio Internacional de Especies de Flora y Fauna Silvestres (CITES 2019), como de Menor Preocupación para la Unión Internacional para la Conservación de la Naturaleza (Cedeño-Vázquez et al. 2012) y en México está incluida en la categoría de Sujeta a Protección Especial por la NOM059- SEMARNAT-2010 (SEMARNAT 2010).

El estado de Tabasco es una zona de distribución natural de $C$. moreletii encontrándolo prácticamente en todo el estado (Platt et al. 2010, Sigler y Gallegos 2017). Diferentes estudios se han realizado para la especie en esta región, sobre manejo, reproducción y crianza en cautiverio (Barrios-Quiroz y Casas-Andreu 2010, Casas-Andreu et al. 2011), uso y contaminación de hábitat (Córdova-Carrillo et al. 2010, Rueda et al. 2017), antropológicos (Erandeni 2009, Lorente 2018) y ecología reproductiva (LópezLuna et al. 2015, López-Luna et al. 2020). En el caso de estudios de estimación poblacional para Tabasco únicamente se cuenta con información reciente del Programa de Monitoreo del Cocodrilo de
Pantano Crocodylus moreletii, para México, Belice y Guatemala realizada en cuatro sitios (Rivera-Téllez et al. 2017).

La Reserva de la Biósfera Pantanos de Centla (RBPC) localizada al noroeste del estado de Tabasco, es el segundo sistema más importante de humedales de agua dulce con influencia marina en América Latina debido a su productividad biológica (BarbaMacías et al. 2014), es considerado sitio Ramsar y forma parte del corredor Biológico Mesoamericano (Álvarez 2013). El cocodrilo de pantano es una de las especies que habita en este humedal (RodríguezQuevedo et al. 2010) y representa un importante recurso para los habitantes, por lo que resulta necesario conocer el estado poblacional de $C$. moreletii en esta área que permita establecer acciones de conservación y manejo para la especie. La cuantificación de los cocodrilos en ambientes naturales representa información básica para las estrategias de manejo y conservación (Cupul-Magaña 2009). Por lo anterior el presente trabajo tuvo como objetivo evaluar la abundancia y estructura poblacional de la especie en cuatro localidades en la Reserva de la Biósfera Pantanos de Centla.

\section{MATERIALES Y MÉTODOS}

\section{Área de estudio}

El estudio se llevó a cabo en la Reserva de la Biósfera Pantanos de Centla localizada en la delta de los ríos Usumacinta y Grijalva, al noreste del estado de Tabasco en la provincia fisiográfica Llanura Costera del Golfo Sur ( $17^{\circ} 57^{\prime} 53^{\prime \prime}$ LN y $92^{\circ} 06^{\prime} 39^{\prime \prime}$ LO; $18^{\circ} 39^{\prime} 03^{\prime \prime}$ LN y $92^{\circ} 47^{\prime} 58^{\prime \prime}$ LO) (Figura 1). La RBPC tiene una superficie de 302706 ha, que incluye parte de los municipios de Centla, Jonuta y Macuspana, cuenta con dos zonas núcleo y una de amortiguamiento (INE 2000). En la región se presentan tres tipos de climas de acuerdo a la clasificación de Köpen modificada por García (1988): Tropical húmedo con lluvias todo el año $(A f)$, Tropical húmedo con una estación seca corta $(A m)$ y Tropical subhúmedo con una estación seca de cuatro a seis meses $(A w)$, con una temperatura media anual de $26^{\circ} \mathrm{C}$, una precipitación promedio de $1600 \mathrm{~mm}$ y un 

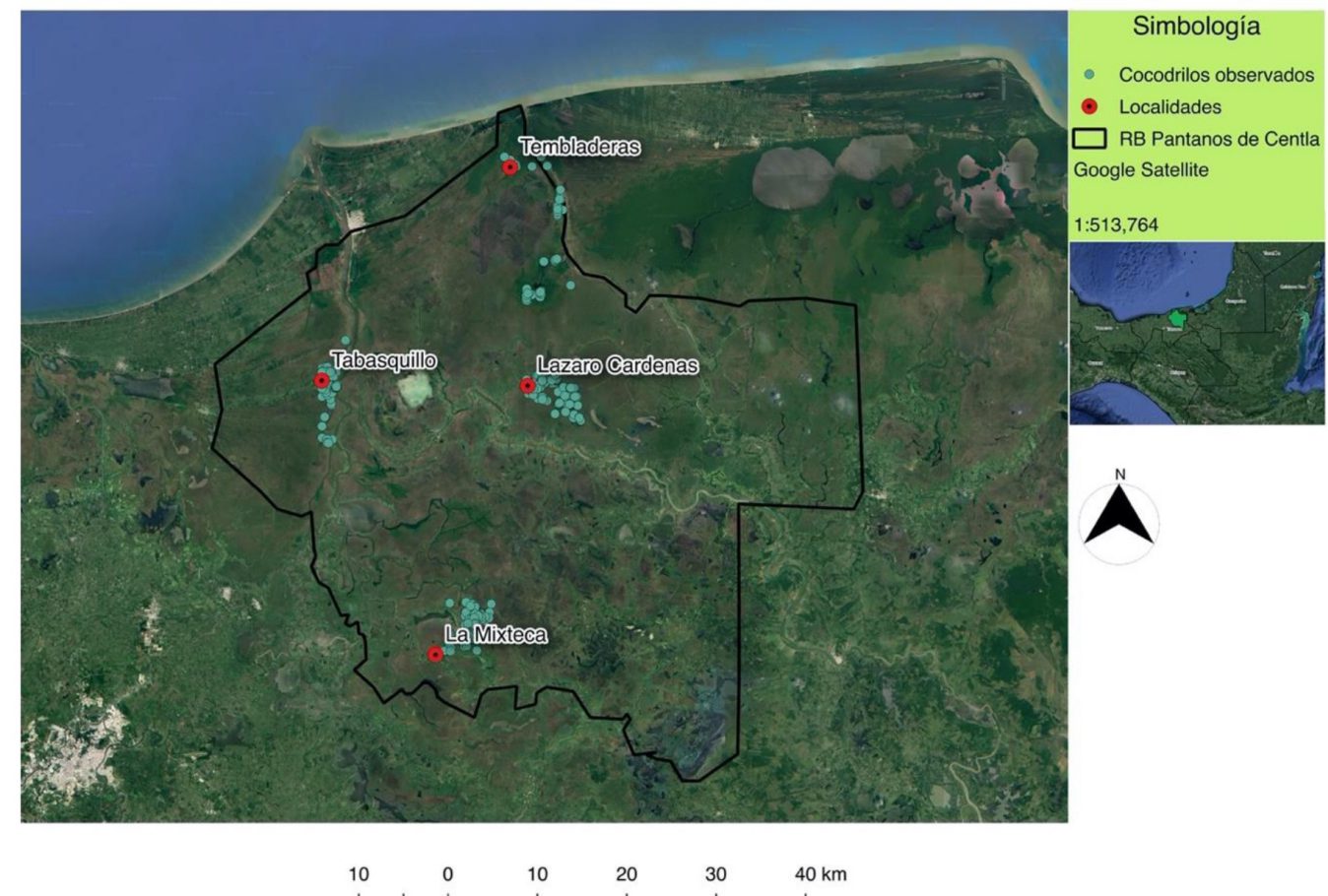

Figura 1. Mapa del área de estudio, se presentan las cuatro localidades y los puntos de registro de los cocodrilos. Escala: $1: 513,764$

rango altitudinal de -1 a 7 msnm (INE 2000, BarbaMacías et al. 2015).

En la RBPC se han diferenciado las siguientes asociaciones vegetales: pukteal o selva mediana subperennifolia de puckteales (Bucida buceras); tintales o selva baja subperennifolia de tinto (Haematoxylum campechianum); manglar (Rhizophora mangle); mucal o matorral de Dalbergia brownii; palmar de Acoelorraphe wrightii; guanal o palmar de Sabal mexicana y asociaciones de vegetación hidrófila (Guadarrama-Olivera y Ortiz-Gil 2000, Romero et al. 2000, López-Jiménez 2019). La diversidad faunística, se compone de 52 especies de peces, 27 de anfibios, 68 de reptiles, 104 de mamíferos y 255 de aves (Romero et al. 2000). Las actividades económicas predominantes son de origen primario como agricultura, ganadería bovina y pesca (Barba-Macías et al. 2014).

\section{Método empleado}

El presente trabajo deriva del financiamiento otorgado del Progama de Conservación de Especies en Riesgo (PROCER), por lo que su realización se limitó al tiempo de duración entre los meses de septiembre a diciembre de 2018. Mensualmente se llevó a cabo un recorrido en cada una de las cuatro diferentes localidades: Tabasquillo ( $18^{\circ} 22^{\prime} 25^{\prime \prime} \mathrm{LN}$, $92^{\circ} 40^{\prime} 59 "$ LO), Tembladeras ( $18^{\circ} 35^{\prime} 21^{\prime \prime}$ LN, $92^{\circ} 28^{\prime}$ 59" LO), La Mixteca ( $18^{\circ} 05^{\prime} 46^{\prime \prime}$ LN, $92^{\circ} 33^{\prime} 48^{\prime \prime}$ LO) y Lázaro Cárdenas ( $18^{\circ} 22^{\prime}$ 05" LN, $92^{\circ} 27^{\prime}$ 54" LO) (Figura 1), para realizar conteos nocturnos de cocodrilos. Las localidades fueron elegidas en función a su distribución a lo largo de la RBPC, disponibilidad de pobladores en participar en el trabajo, seguridad para los investigadores y accesibilidad de los sitios. En cada localidad se estableció un transecto fijo con diferentes longitudes y amplitud de los cuerpos de agua, tomando como referencia a los ríos y lagunas navegables. Cada recorrido nocturno se llevó a cabo en fase de luna nueva y se utilizó una lancha de $4 \mathrm{~m}$ de eslora con motor fuera de borda de $15 \mathrm{HP}$, navegando a una velocidad constante de $10 \mathrm{~km} \mathrm{~h}^{-1}$ (Magnusson 1982, Fakuda et al. 2013). Los muestreos iniciaron a las $21: 00 \mathrm{~h}$ en oscuridad total y en promedio 
tardaron $3.5 \mathrm{~h}$.

El conteo y estimación de tallas de los cocodrilos fue realizado siguiendo el método descrito por Chabreck (1966), el cual consiste en ubicar a los organismos por el destello de la luz de los ojos cuando es alumbrado por una lámpara, lo que lo ciega momentáneamente y permite acercarse: Para ello se empleó una lámpara de hálogeno con haz de luz cerrada con alcance de un millón de candelas en lugares abiertos y una lámpara de mano en lugares cerrados. Por cada recorrido se contabilizaron el número total de cocodrilos y se tomaron los siguientes datos: coordenadas del sitio de avistamiento, hora, fecha, longitud total (LT) estimada y sin datos (SD) cuando no fue posible determinar la LT.

Debido a que no se realizaron capturas de cocodrilos, la estructura poblacional se basó en la estimación de la LT de los individuos, esta se realizó valorando el tamaño de los cocodrilos a simple vista a distancias no mayores a $3 \mathrm{~m}$ de la embarcación hasta donde se encontraba cada ejemplar. Posteriormente se agruparon tomando en cuenta la distribución propuesta por Sánchez et al. (2011) para C. moreletii que considera: Clase I o Cría que son los que sobrevivieron al primer invierno $(<50 \mathrm{~cm})$; Clase II o juveniles (entre 51 a $100 \mathrm{~cm}$ ); Clase III o sub adultos (entre $101 \mathrm{a} 150 \mathrm{~cm}$ ) y Clase IV o adultos (entre 151 a $200 \mathrm{~cm}$ ), adultos grandes o Clase V (> $201 \mathrm{~cm}$ ) y solo ojos $(\mathrm{S} / \mathrm{O})$. Se elaboraron histogramas de frecuencia de las clases de tamaño por localidad de muestreo.

Se estimó la tasa de encuentro (TE) para cada localidad de muestro a partir del número de organismos observados entre la distancia recorrida en kilómetros lineales para cada sitio y se expresó como cocodrilos por kilómetro (King et al. 1994, Cupul-Magaña 2009, Sánchez et al. 2011).

Se utilizó el modelo de Messel et al. (1981) modificado por Thorbjarnarson et al. (2000) para estimar el tamaño poblacional por localidad. El modelo consiste en calcular el valor porcentual de la población observada $(\mathrm{P})$ con la cual se estimó la población total $(\mathrm{N})$. El valor de $\mathrm{P}$ representa el porcentaje de individuos que no pudieron ser observa- dos y se obtuvo de la siguiente forma (Messel et al. 1981):

$$
P=\frac{m}{(2 s+m) 1.05}
$$

Donde: $\mathrm{P}$ es el porcentaje de la población observada por sitio, $m$ es la media del número de cocodrilos observados por sitio, $s$ es la desviación estándar del número de cocodrilos observados por sitio y el nivel de error es de 1.05.

El cálculo de la población total $(\mathrm{N})$ con un límite de confianza del 95\%, aceptando la normalidad de los datos se obtuvo mediante la fórmula (Messel et al. 1981):

$$
N=\frac{m}{P} \pm \frac{[1.96(s)]^{1 / 2}}{P}
$$

Para determinar diferencias significativas entre el número de individuos encontrados por localidad se aplicó la prueba no paramétrica de Kruskal Wallis y el test de Dunn como prueba de comparación múltiple (Dunn, 1964). Se consideró un nivel de significancia $<0.05$

\section{RESULTADOS}

En total se obtuvieron 797 registros de cocodrilos en 16 conteos nocturnos, donde se recorrieron un total de $372 \mathrm{~km}$ que correspondieron a $76 \mathrm{~km}$ en La Mixteca, 80 km Lázaro Cárdenas, 92 km en Tabasquillo y $124 \mathrm{~km}$ en Tembladeras. Las mayores tasas de encuentro y abundancia poblacional se registraron en las localidades de La Mixteca (3.14 ind $\mathrm{km}^{-1}$ lineal y $84.79 \pm 6.44$ individuos) y Lázaro Cárdenas (2.94 ind $\mathrm{km}^{-1}$ lineal y $75.66 \pm 4.65$ individuos); en tanto Tabasquillo (2.00 ind $\mathrm{km}^{-1}$ lineal y $59.80 \pm 4.26$ individuos) y Tembladeras (1.12 ind $\mathrm{km}^{-1}$ lineal y $54.09 \pm 6.31$ individuos) presentaron las cantidades más bajas (Tabla 1). La prueba de Kuskal-Wallis mostró diferencias significativas entre las medianas de los individuos observados en cada transecto $(H=10.775$, gl. $=3, p<0.05)$. El test de Dunn a posteriori mostró diferencias significativas entre las localidades La Mixteca - Tembladeras y La Mixteca - Lázaro Cárdenas. 
Tabla 1. Tamaño poblacional de Crocodylus moreletii y Tasa de Encuentro en cuatro localidades de la RBPC. $m=$ valor promedio de número de cocodrilos observados por sitio; $s=$ desviación estándar; $p=$ porcentaje de la población observada por sitio, $\mathrm{N}$ = tamaño estimado de la población por sitio; TE = tasa de encuentro.

\begin{tabular}{lccccccc}
\hline Población & Longitud del transecto $(\mathrm{km})$ & $(\mathrm{m})$ & $(\mathrm{s})$ & $(\mathrm{P})$ & $(\mathrm{N})$ & Intervalo & TE $($ Ind./km lineal $)$ \\
\hline La Mixteca & 19 & 59.75 & 10.50 & 0.70 & 84.79 & $78.35-91.22$ & 3.14 \\
Lázaro Cárdenas & 20 & 58.75 & 6.65 & 0.78 & 75.66 & $71.01-80.31$ & 2.94 \\
Tabasquillo & 23 & 46.00 & 5.48 & 0.77 & 59.80 & $55.54-64.06$ & 2.00 \\
Tembladeras & 31 & 34.75 & 8.38 & 0.64 & 54.09 & $47.78-60.40$ & 1.12 \\
\hline
\end{tabular}

De los 797 registros obtenidos fue posible aproximar la LT de 362 cocodrilos: 195 clase I, 118 clase II, 10 clase III, 23 clase IV, 16 clase V y 435 $\mathrm{S} / \mathrm{O}$. Por localidad se determinaron la LT de 89 individuos en La Mixteca, 82 en Lázaro Cárdenas, 96 en Tabasquillo y 95 Tembladeras. Con respecto a la estructura poblacional, aunque se observa a todas las clases de tamaño en los cuatro sitios, las poblaciones están mayormente compuestas de individuos jóvenes (clases I y II) (Figura 2). Los mayores registros para la clase I se obtuvieron en las localidades de Lázaro Cárdenas y Tabasquillo, para la clase II en Tembladeras y La Mixteca, en tanto que las clases IV y $\mathrm{V}$ el mayor número de registros se presentó en La Mixteca en donde se observaron 30 individuos, mientras que entre las otras tres localidades se registraron únicamente nueve individuos en total (Figura 3).

\section{DISCUSIÓN}

La TE es un índice basado en el número de cocodrilos observados sobre la distancia recorrida (esfuerzo de muestreo) que provee una estimación de la abundancia relativa, debido a que por diversos factores ambientales como lluvia, temperatura y nivel del agua y físicas como la vegetación circundante, ancho y forma del cuerpo de agua no es posible observar a todos los individuos presentes (García-Grajales et al. 2007, Sánchez et al. 2011). Este índice ha sido utilizado ampliamente en los estudios poblacionales sobre cocodrilos en el mundo (González et al. 2018), ya que se basa en el suposición que su relación y el tamaño de la población local se mantiene comparativamente constante entre muestreos y que cualquier variación reflejará un cambio proporcional en la población real (Platt y Thorbjarnarson 2000), además que permite estable- cer comparaciones entre diferentes sitios y temporadas (García-Grajales y Buenrostro-Silva 2017). Las menores TE presentadas en las localidades de Tembladeras y Tabasquillo pudieron deberse a que la localidad de Tembladeras presenta un alta densidad de manglares que persiste por toda la zona lo que permite a los cocodrilos refugiarse dificultando su observación, en tanto en la localidad de Tabasquillo se pudo observar una mayor presión humana por ganadería bovina.

Al comparar las TE obtenidas en Laguna del Carpintero, Tamaulipas (27.5 ind $\mathrm{km}^{-1}$; Villegas y Reynoso 2013) y Laguna Esmeralda, Quintana Roo (44.4 ind $\mathrm{km}^{-1}$; Cedeño y Pérez 2010), las TE registradas en el presente trabajo se encuentran por debajo; pero superior a lo reportado en Área de Protección de Flora y Fauna Laguna de Términos (APFFLT), Campeche (0.69 ind $\mathrm{km}^{-1}$, Rodas-Trejo et al. 2017) y en canales costeros en la Reserva de La Biósfera Los Petenes, Campeche (0.47 ind $\mathrm{km}^{-1}$, Padilla et al. 2010); y similar a la TE nacional obtenida en el Programa de monitoreo del cocodrilo de pantano (Crocodylus moreletii) México-BeliceGuatemala, (2011-2015) (3.23 ind km ${ }^{-1}$, Muñiz y López-Segurajáuregui 2018).

Con respecto al cálculo de las abundancias de poblaciones de cocodrilos a través de la estimación de la fracción visible de la población (Messel et al. 1981), existen pocos trabajos realizados en México (García-Grajales y Buenrostro-Silva 2017), la mayoría para Crocodylus acutus (Brandon-Pliego 2007, Hernández-Hurtado et al. 2011, GarcíaGrajales y Buenrostro-Silva 2014, García-Grajales y Buenrostro-Silva 2017); en tanto que para C. moreletii únicamente se encontró el reportado por Rodas-Trejo et al. (2017). Para RBPC solo hay datos de abundancia del año 1990 en la cual se estimó una población 


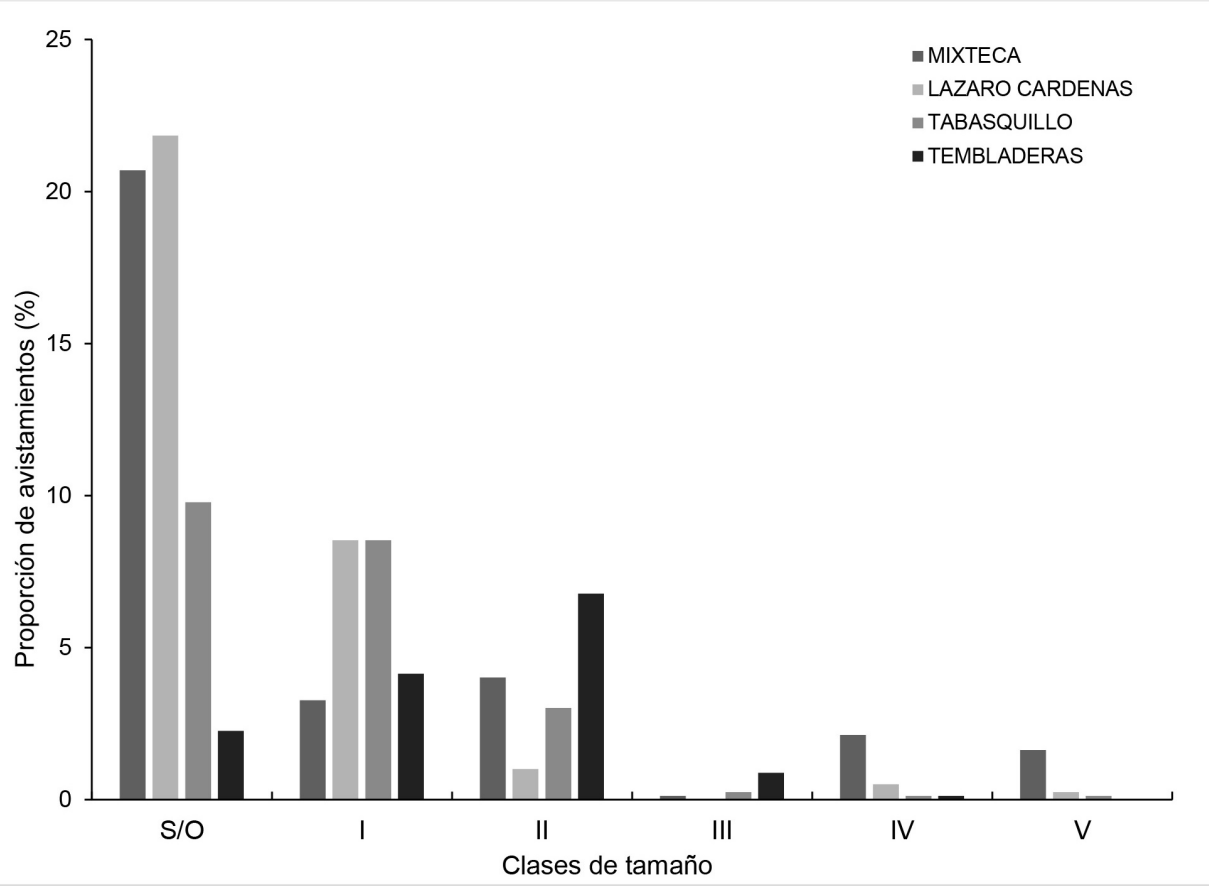

Figura 2. Proporción de avistamientos por clases de tamaño en las cuatro localidades en la Reserva de La Biósfera Pantanos de Centla.

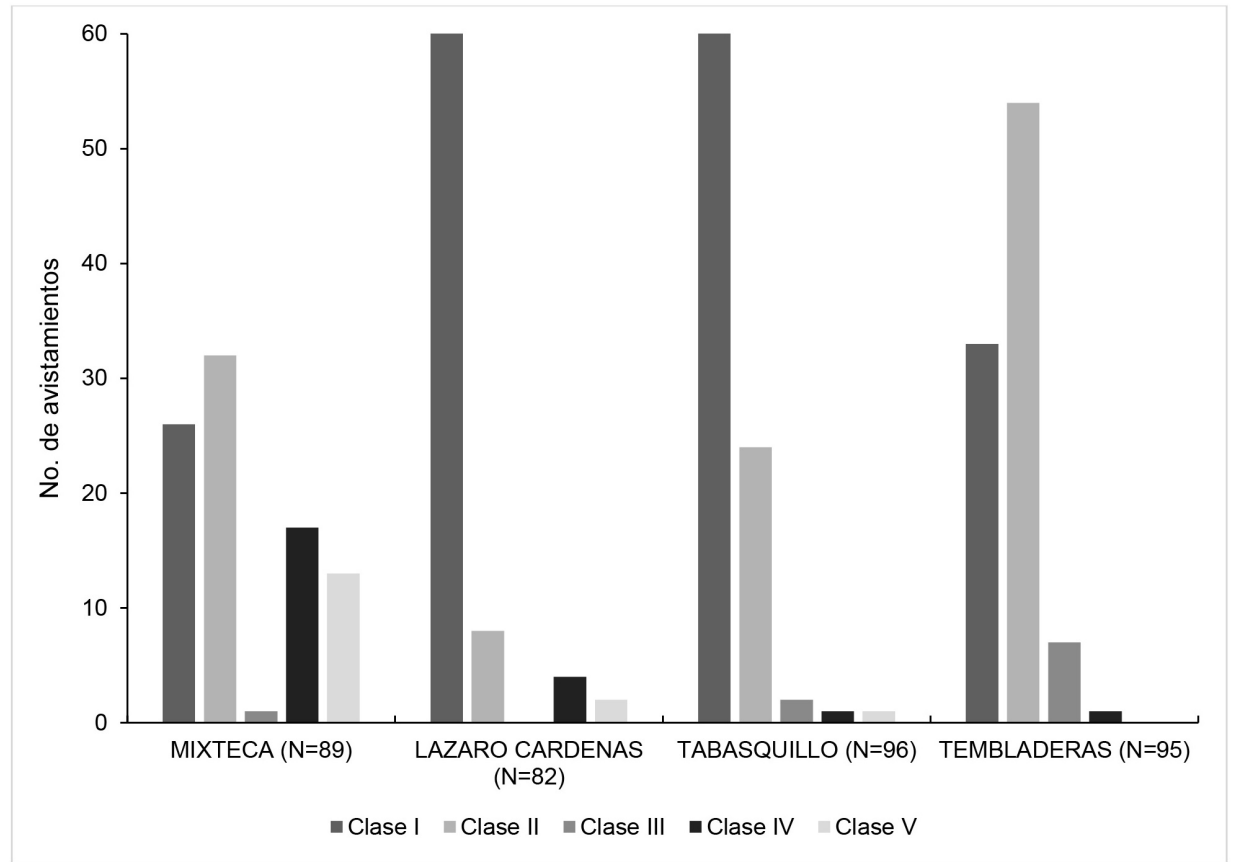

Figura 3. Estructura poblacional de Crocodylus moreletii por clase de tamaño en cuatro localidades en la Reserva de la Biósfera Pantanos de Centla. 
de 1000 cocodrilos de pantano en $221 \mathrm{~km}^{2}$ (Romeu 1998). Si bien estas estimaciones presentan un panorama del estatus de las poblaciones locales de cocodrilos, al realizar comparaciones de abundancias poblacionales entre sitios es importante considerar factores que puedan afectarlas, como las longitudes de los transectos, disponibilidad de sitios de anidación, cantidad y tipo de vegetación circundante, presión antropogénica, características de los cuerpos de agua (corriente, profundidad, salinidad) y temporalidad del muestro (Platt y Thorbjarnarson 2000).

La estructura poblacional de los cocodrilos se detalla en función de la proporción de sexos y la distribución de la clasificación de longitud corporal de cada uno de los individuos de la población y se construye mediante la proporción de clases de los individuos observados (González et al. 2018). El seguimiento de estos parámetros nos permite conocer el estado de conservación a través del tiempo en un área determinada (Ojasti y Dallmeier 2000). La estructura general de la población de cocodrilos en las cuatro localidades, indica una mayor proporción de individuos de las clases I (53.87\%), II $(32.60 \%)$ y III (2.76) coincidente con la estructura reportada a nivel nacional en la que las primeras tres tallas representan el $81 \%$ de los avistamientos (Rivera-Téllez et al. 2017). Por otro lado, esta estructura varía con lo reportado por Cedeño y Pérez (2010) en la Laguna Esmeralda en Quintana Roo y por Rodas et al. (2017) en el APFFLT, en donde las clases III y IV se registraron en mayor proporción pudiendo deberse al mayor tiempo de muestro lo que incrementa las probabilidades de observación de individuos. Por otro lado, el registro de una mayor proporción de cocodrilos de las clases I y II en el presente trabajo, pudo deberse entre otros factores a la temporalidad del muestreo ya que estos se realizaron posteriores a la época de nacimientos lo que permite la mayor presencia de clases bajas. Otra causa se puede adjudicar a que únicamente se pudo determinar la LT en el $45 \%$ de los avistamientos, ya que al sentir la presencia del bote los cocodrilos se sumergían, comportamiento evasivo documentado en individuos subadultos y adultos (Kushlan y Mazzotti 1986). En cuanto al estado de conservación de $C$. moreletii en las cuatro localidades, se puede vislumbrar que si bien las TE son bajas principalmente en las localidades de Tabasquillo y Tembladeras, la estructura poblacional representada por individuos de todas las clases con mayor abundancia de la clase I en todas las localidades, nos permite inferir que existen las condiciones para la reproducción denotando cierta estabilidad.

Si bien, los datos presentados se refieren a un muestreo de cuatro meses y pueden estar subestimados, la importancia del presente trabajo radica en que es una de las primeras aproximaciones del estatus poblacional de $C$. moreletii en localidades de la Reserva de la Biósfera Pantanos de Centla. Se considera necesario por la importancia de esta área natural protegida como sitio de presencia y reproducción de $C$. moreletii que se continúe con el muestro en otras épocas del año y ampliar el número de localidades que permitan poder establecer una tendencia de las poblaciones locales para construir una línea base de información a nivel de toda la Reserva de la Biósfera Pantanos de Centla.

\section{CONCLUSIONES}

La abundancia y estructura poblacional reportada representa la primera aproximación poblacional para C. moreletii en la RBPC desde 1990. En comparación con otros estudios los datos obtenidos se encuentran en un rango intermedio y similar a los datos obtenidos en el Programa de monitoreo de México-Belice-Guatemala. La presencia de individuos de todas las clases de tamaño, la mayor proporción de avistamientos de las clases I y II, así como el registro de individuos adultos en las cuatro localidades de la RBPC, denotan poblaciones en crecimiento y pueden considerarse en óptimas condiciones. Es necesario continuar con estimaciones poblacionales en diferentes épocas del año y sitios de la RBPC para detectar tendencias. También se recomienda realizar estudios que incluyan componentes sociales, calidad del hábitat y ecológicos como anidaciones, con la finalidad de proponer estrategias de conservación y manejo de la especie 
como puede el aprovechamiento sustentable bajo el esquema de rancheo.

\section{AGRADECIMIENTOS}

A la Dirección de La Reserva de Biósfera Pantanos de Centla de la Comisión Nacional de
Áreas Naturales Protegidas y al Programa de Conservación de Especies en Riesgo (PROCER), por el financiamiento otorgado para la ejecución del trabajo. A los integrantes del Programa de Manejo de Áreas Protegidas (PROMANP) de las localidades de Tabasquillo, Tembladeras, La Mixteca y Lázaro Cárdenas por su acompañamiento y guía en las actividades.

\section{LITERATURA CITADA}

Álvarez del Toro M (1974) Los Crocodylia de México (estudio comparativo). Instituto Mexicano de Recursos Naturales Renovables, A.C. México. 70p.

Álvarez IP (2013) Corredor Biológico Mesoamericano en México. Comisión Nacional para el Conocimiento y Uso de la Biodiversidad. Biodiversitas 110: 1-5.

Balaguera-Reina SA, González-Maya JF (2010) Percepciones, conocimiento y relaciones entre los Crocodylia y poblaciones humanas en la Vía Parque Isla de Salamanca y su zona de amortiguamiento, Caribe colombiano. Revista Latinoamericana de Conservación 1: 53-63.

Barba-Macías E, Valadez F, Pinkus M, Pinkus J (2014) Revisión de la problemática socioambiental de la Reserva de la Biósfera Pantanos de Centla, Tabasco. Investigación y Ciencia 60: 51-58.

Barba-Macías E, Valadez F, Pinkus M, Pinkus J, Juárez FJ (2015) Reserva de la Biosfera Pantanos de Centla: Aspectos socio-ambientales prioritarios. En: Ortega-Rubio A, Pinkus-Rendón MJ, Espita-Moreno IC (eds.) Las Áreas Naturales Protegidas y la investigación científica en México. Centro de Investigaciones Biológicas del Noroeste, Universidad Autónoma de Yucatán, Mérida, y Universidad Michoacana de San Nicolás de Hidalgo. México. pp: 11-29.

Barrera BN, Toledo V (2005) Ethnoecology of the Yucatec maya: Symbolism, knowledge and management of natural resources. Journal of Latin American Geography 4: 9-41.

Barrios-Quiroz G, Casas-Andreu G (2010) Crecimiento con diferentes dietas en crías de Crocodylus moreletii Dumeril, Bibron \& Dumeril 1851 (Crocodylia: Crocodylidae) en cautiverio, Tabasco, México. Revista Latinoamericana de Conservación 1: 104-111.

Brandon-Pliego, JD (2007) Estudio poblacional de Crocodylus acutus (Cuvier, 1807) (Reptilia: Crocodylia) en Jamiltepec, Oaxaca. Ciencia y Mar 11: 29-37.

Casas-Andreu G, Barrios-Quiroz G, Macip-Ríos R (2011) Reproducción en cautiverio de Crocodylus moreletii en Tabasco, México. Revista Mexicana de Biodiversidad 82: 261-273.

Chabreck RH (1966) Methods of determining the size and composition of alligators populations in Louisiana. Proceedings 20th Annual Conference Southeastern Association of Game and Fish Commissioners 20: 105112.

Cedeño VJR, Pérez SD (2010) El Cocodrilo de Pantano (Crocodylus moreletii) en Laguna Esmeralda, Quintana Roo, México. Revista Latinoamericana de conservación 1: 91-98.

Cedeño-Vázquez JR, Platt SG, Thorbjarnarson J (2012) Crocodylus moreletii. The IUCN Red List of Threatened Species 2012: e.T5663A3045579. https://dx.doi.org/10.2305/IUCN.UK.2012.RLTS.T5663A3045579.en. Fecha de consulta: 15 de marzo 2020. 
CITES (2019) Apéndices I, II y III. Comercio Internacional de Especies de Flora y Fauna Silvestres. 80p. https://www.cites.org/esp/app/appendices.php. Fecha consultada: 25 de mayo de 2020.

Córdova-Carrillo A, Pérez-Sánchez E, Rodríguez-Quevedo F, Ovando-Hidalgo N, Zequeira-Larios C (2010) Agroquímicos utilizados en la Reserva de la Biosfera Pantanos de Centla: Una afectación indirecta para el Cocodrilo de Pantano (Crocodylus moreletii). KUXULKAB' 17: 31-42.

Cupul-Magaña FG (2003) Cocodrilo: medicina para el alma y el cuerpo. Revista Biomédica 14: 45-48.

Cupul-Magaña FG (2009) ¡A contar cocodrilos! Comentarios básicos sobre algunos métodos para evaluar poblaciones silvestres. Ciencia y Mar 13: 3-14.

Dunn O (1964) Multiple Comparisons Using Rank Sums. Technometrics 6: 241-241

Erandeni AOT (2009) Entre santos y cocodrilos. Acercamiento a dos festividades en Tabasco y Guatemala. Península 4: 117-131.

Fukuda Y, Saalfeld K, Webb G, Manolis C, Risk R (2013) Standardised method of spotlight surveys for crocodiles in the tidal rivers of the Northern Territory, Australia. Northern Territory Naturalist 24:14-32.

García E (1988) Modificaciones al sistema de clasificación climática de Köpen, para adaptarlo a las condiciones de la República Mexicana. Offset Larios. México. 98p.

García-Grajales J, Buenrostro-Silva A (2014) Abundancia y estructura poblacional de Crocodylus acutus (Reptilia: Crocodylidae) en la laguna Palmasola, Oaxaca, México. Revista de Biología Tropical 62: 165-172.

García-Grajales J, Buenrostro-Silva A (2017) Estimación poblacional del cocodrilo americano (Crocodylus acutus) en el Parque Nacional Lagunas de Chacahua, Oaxaca, México. Revista Mexicana de Biodiversidad 88: 936-943.

García-Grajales J, Buenrostro-Silva A, Escobedo-Galván A (2007) Análisis de los métodos usados para estimar la abundancia de las poblaciones silvestres de cocodrilianos (Crocodylia) en México. Ciencia y Mar 31: 23-32.

González JM, López LMA, Padilla ES, Barrios QG (2018) Monitoreo poblacional como base para el rancheo. En: Barrios G, Cremieux J (Eds). Protocolo de rancheo para cocodrilo de pantano (Crocodylus moreletii) en México. Comisión Nacional para el Conocimiento y Uso de la Biodiversidad. México. pp: 28-38.

Guadarrama-Olivera y Ortiz-Gil (2000) Análisis de la flora de la Reserva de la Biosfera de los Pantanos de Centla, Tabasco, México. Universidad y Ciencia 15: 67-104.

Hernández-Hurtado H, Romero-Villarruel JJ, Hernández-Hurtado PS (2011) Ecología poblacional de Crocodylus acutus en los sistemas estuarinos de San Blas, Nayarit, México. Revista Mexicana de Biodiversidad 82: 887-895.

INE (2000) Programa de Manejo de la Reserva de la Biósfera Pantanos de Centla. México. 222p.

King F, Hutton J, Manolis C, Miller J, Jelden D, Mc-Namara K, Rodríguez M, Ross JP, Saalfeld K, Velasco A, Webb GJ, Woodward A (1994) Guidelines on monitoring crocodilian populations. In: Proceedings 2nd Regional Meeting (Eastern Asia, Oceania and Australasia) of the Crocodile Specialist Group, IUCN, Gland, Switzerland. pp: 1-3.

Kushlan JA, Mazzotti FJ (1986) Population biology and status of the American Crocodile in South Florida. En: Proceedings of the 7th Working Meeting of the Crocodile Specialist Group. IUCN, Gland, Switzerland. pp: 184-206.

López-Jiménez LN (2019) Tabasco es el edén de los humedales. KUXULKAB' 25: 39-51. 
López-Luna MA, Hidalgo-Miharta MG, Aguirre-León G, González-Ramón M, Rangel-Mendoza JA (2015) Effect of nesting environment on incubation temperature and hatching success of Morelet's crocodile (Crocodylus moreletii) in an urban lake of Southeastern Mexico. Journal of Thermal Biology 49: 66-73.

López-Luna MA, González-Soberano J, González-Jáuregui M, Escobedo-Galván AH, Suárez-Domínguez EA, Rangel-Mendoza JA, Morales-Mávila JE (2020) Nest-site selection and nest size influence the incubation temperature of Morelet's crocodiles. Journal of Thermal Biology 102624. DOI: 10.1016/j.jtherbio.2020.1026 24

López-Luna MA, Rodríguez AG, González MCR, Ramos TR (2019) Estudio de caso: Los cocodrilos de Villahermosa. En: Cruz AA, Cruz MJ, Valero JP, Rodríguez RFP, Melgarejo ED, Mata ZEE, Palma LDJ (eds). La biodiversidad en Tabasco. Estudio de Estado, vol 2, Ciudad de México. pp: 382-385.

Lorente FD (2018) Pejelagartos, cocodrilos y canoas. De los seres del agua bajo el dominio de Ix Bolon entre los mayas chontales de Tabasco. Anales de Antropología 52: 179-195.

Magnusson WE (1982) Techniques of surveying for crocodilians. In: Crocodiles: Proceedings of the 5th Annual Working Meeting of the Crocodile Specialist Group of the Species Survival Commission of IUCN-The World Conservation Union. Gland, Switzerland. pp: 389-403.

Messel H, Vorlicek GC, Wells AG, Green WJ (1981) Surveys oftidal river systems in Northern Territory of Australia and their crocodilepopulations. Monographs 1. Sydney: Pergamon Press. Australia. 466p.

Muñiz CM y López-Segurajáuregui G (2018) Antecedentes. En: Barrios G, Cremieux J (Eds). Protocolo de rancheo para cocodrilo de pantano (Crocodylus moreletii) en México. Comisión Nacional para el Conocimiento y Uso de la Biodiversidad. México. pp: 16-25.

Ojasti J, Dallmeier F (2000) Manejo de fauna Silvestre neotropical. Smithsonian Institution, MAB Biodiversity Program, Washington, DC. USA. 233p.

Padilla SE, Perera ET, González MJ, Gómez JOD (2010) Estudio de caso: el cocodrilo de pantano Crocodylus moreletii en la Reserva de la Biosfera Los Petenes, Campeche. En: Villalobos-Zapata GJ, Mendoza JV (eds). La Biodiversidad en Campeche: Estudio de Estado. Comisión Nacional para el Conocimiento y Uso de la Biodiversidad (CONABIO), Gobierno del Estado de Campeche, Universidad Autónoma de Campeche, El Colegio de la Frontera Sur. México. pp: 344-348.

Platt SG, Sigler L, Rainwater TR (2010) Morelet's Crocodile Crocodylus moreletii. En: Manolis SC, Stevenson C (eds). Crocodiles. Status survey and conservation action plan. 3rd Ed. Crocodile Specialist Group Darwin. Australia. pp. 79-83.

Platt SG, Thorbjarnarson JB (2000) Population status and conservation of Morelet's crocodile Crocodylus moreletii, in northern Belize. Biological Conservation 96: 13-20.

Rivera-Téllez E, López SG, Antaño DLA, Beniítez DH (2017) Informe del Programa de Monitoreo del Cocodrilo de Pantano en México, temporadas 2014 a 2015 y análisis de tendencias del 2011 al 2015. Comisión Nacional para el Conocimiento y Uso de la Biodiversidad. México. 35p.

Rodas-Trejo J, Ocampo-González P, Hernández-Nava J, Mandujano-Camacho H, Coutiño-Hernández PR, Orantes-Zebadua MA (2018) Percepción, conocimiento popular y aprovechamiento hacia el cocodrilo de pantano (Crocodylus moreletii Duméril \& Bibron) por pobladores del área de protección de flora y fauna Laguna de Términos, Campeche, México. Agroproductividad 11: 45-50. 
Rodas-Trejo J, Ocampo-González P, Mandujano-Camacho H, Grajales-Zepeda R, Hernández-Nava J, (2017) Estado poblacional del Crocodylus moreletii en el Área Natural Protegida Laguna de Términos, Campeche, México. Quehacer científico en Chiapas 12: 41-46.

Rodríguez-Quevedo F, Pérez E, Córdova-Carrillo A, Ovando-Hidalgo N, García-Ulloa F (2010) Morelet’s crocodile in the Biosphere Preserve of Centla Swamps, Tabasco, Mexico. Crocodile Specialist Group Newsletter 29: 11-13.

Romero JC, García A, Bautista CA, Pérez PH (2000) Caracterización de la Reserva de la Biosfera Pantanos de Centla. Universidad y Ciencia 15: 15-28.

Romeu E (1998) Cocodrilos mexicanos. Biodiversitas 4: 1-8.

Rueda C, López LMA, Olivera LDG (2017) Uso de hábitat del cocodrilo de pantano Crocodylus moreletii en una laguna urbanizada en México. Quehacer Científico en Chiapas 12: 35-40.

Sánchez HO, López GS, García ANOH, Benítez HD (2011) Programa de monitoreo del Cocodrilo de pantano (Crocodylus moreletii) México-Belice-Guatemala. México. Comisión Nacional para el Conocimiento y Uso de la Biodiversidad. México. 270p.

SEMARNAT (2010) Norma Oficial Mexicana NOM-059- SEMARNAT-2010, que determina las especies de flora y fauna silvestres terrestres y acuáticas, endémicas, amenazadas, en peligro de extinción y sujetas a protección especial. México. Órgano del Gobierno Constitucional de los Estados, Gobierno Federal. 103p

Sigler L, Gallegos MJ (2017) El conocimiento sobre el cocodrilo de Morelet Crocodylus moreletii (Duméril y Duméril 1851) en México, Belice y Guatemala. The Dallas World Aquarium. México. 216p.

Thorbjarnarson JT, Platt S, Saw-Tun-Khaing U (2000) A population survey of the estuarine crocodile in the Ayeryarwady Delta, Myanmar. Oryx 34: 317-324.

Villegas A, Reynoso V (2013) Relative abundance and Habitat Preference in isolated Populations of Morelet's crocodile (Crocodylus moreletii) along the coast of the Gulf of Mexico. Herpetological Conservation and Biology 8: 571-580. 